\title{
DISCOVERY OF TeV GAMMA-RAY EMISSION FROM THE CYGNUS REGION OF THE GALAXY
}

\author{
A. A. Abdo, ${ }^{1}$ B. Allen, ${ }^{2}$ D. Berley, ${ }^{3}$ E. Blaufuss, ${ }^{3}$ S. Casanova, ${ }^{4}$ C. Chen, ${ }^{2}$ D. G. Coyne ${ }^{5}$ R. S. Delay, ${ }^{2}$ B. L. Dingus, ${ }^{4}$ \\ R. W. Ellsworth, ${ }^{6}$ L. Fleysher, ${ }^{7}$ R. Fleysher, ${ }^{7}$ I. Gebauer, ${ }^{1,8}$ M. M. Gonzalez, ${ }^{9}$ J. A. Goodman, ${ }^{3}$ E. Hays, ${ }^{3}$ \\ C. M. Hoffman, ${ }^{4}$ B. E. Kolterman, ${ }^{7}$ L. A. Kelley, ${ }^{5}$ C. P. Lansdell,${ }^{3}$ J. T. Linnemann, ${ }^{1}$ J. E. McEnery, ${ }^{10}$ A. I. Mincer, ${ }^{7}$ \\ I. V. Moskalenko, ${ }^{11}$ P. Nemethy, ${ }^{7}$ D. Noyes, ${ }^{3}$ J. M. Ryan, ${ }^{12}$ F. W. Samuelson, ${ }^{4}$ P. M. Saz Parkinson, ${ }^{5}$ \\ M. Schneider, ${ }^{5}$ A. Shoup,${ }^{13}$ G. Sinnis, ${ }^{4}$ A. J. Smith, ${ }^{3}$ A. W. Strong, ${ }^{14}$ G. W. Sullivan, ${ }^{3}$ V. Vasileiou, ${ }^{3}$ G. P. Walker, ${ }^{4}$ \\ D. A. Williams,${ }^{5}$ X. W. Xu, ${ }^{4}$ AND G. B. YodH ${ }^{2}$ \\ Received 2006 November 20; accepted 2007 February 8; published 2007 February 22
}

\begin{abstract}
The diffuse gamma radiation arising from the interaction of cosmic-ray particles with matter and radiation in the Galaxy is one of the few probes available to study the origin of the cosmic rays. Data from the Milagro gamma-ray observatory - a water Cerenkov detector that continuously views $\sim 2$ sr of the overhead sky-shows that the brightest extended region in the entire northern sky is the Cygnus region of the Galactic plane. The TeV image of the Cygnus region contains at least one new source, MGRO J2019+37, which is $10.9 \sigma$ above the isotropic background, as well as correlations with the matter density in the region. However, the gamma-ray flux from the Cygnus region (after excluding MGRO J2019+37) as measured at $\sim 12 \mathrm{TeV}$ exceeds that predicted from a model of cosmic-ray production and propagation. This observation indicates the existence of either hardspectrum cosmic-ray sources and/or unresolved sources of $\mathrm{TeV}$ gamma rays in the region.
\end{abstract}

Subject headings: acceleration of particles — cosmic rays - gamma rays: observations pulsars: individual $(\mathrm{G} 75.2+0.1)$

\section{INTRODUCTION}

A diffuse gamma-ray flux is expected from the plane of the Galaxy due to cosmic-ray interactions with matter and radiation fields. At energies above $1 \mathrm{GeV}$, the flux from the Galactic plane, as determined from EGRET observations, exceeded predictions based on the matter density measured in the region and the cosmicray spectrum and intensity measured at Earth (Hunter et al. 1997; Strong et al. 2004a, 2004b). Explanations of this excess include unresolved sources, a varying cosmic-ray spectrum or intensity across the Galaxy (Strong et al. 2004a, 2004b; Gralewicz et al. 1997), a hard electron spectrum leading to an increase in the inverse Compton component (Porter \& Protheroe 1997), or the addition of a new production mechanism such as the annihilation of relic dark matter particles (de Boer et al. 2005).

At higher energies, $\mathrm{TeV}$ emission from the Galactic plane was discovered with the data from the Milagro gamma-ray observatory (Atkins et al. 2005). The Milagro flux measurement has been argued to also be in excess of the predicted diffuse gamma radiation (Prodanović et al. 2007). Additionally, the HESS telescope has observed gamma rays arising from the interactions of cosmic rays with molecular clouds near the Galactic center (Aharonian et al. 2006a). The required cosmic-

\footnotetext{
${ }^{1}$ Michigan State University, East Lansing, MI.

${ }^{2}$ University of California, Irvine, CA.

${ }^{3}$ University of Maryland, College Park, MD.

${ }^{4}$ Los Alamos National Laboratory, Los Alamos, NM.

${ }^{5}$ University of California, Santa Cruz, CA.

${ }^{6}$ George Mason University, Fairfax, VA.

${ }^{7}$ New York University, New York, NY.

${ }^{8}$ Universität Karlsruhe, Insitute für Experimentelle Kernphysik, Karlsruhe, Germany.

${ }^{9}$ Instituto de Astronomia, Universidad Nacional Autonoma de Mexico, DF, México.

${ }^{10}$ NASA Goddard Space Flight Center, Greenbelt, MD.

${ }^{11}$ Stanford University, Stanford, CA.

${ }^{12}$ University of New Hampshire, Durham, NH.

${ }^{13}$ Ohio State University, Lima, OH.

${ }^{14}$ Max-Planck-Institut für extraterrestrische Physik, Garching, Germany.
}

ray flux is a factor of 3-9 times higher, and the spectrum is harder than the locally measured cosmic-ray spectrum and intensity, supporting the hypothesis that the cosmic-ray spectrum depends on the location in the Galaxy.

The strongest excess in the previously reported Milagro diffuse flux was near Galactic longitude $80^{\circ}$ - the Cygnus region. This region of the Galaxy is a natural laboratory for the study of cosmicray origins. It contains a large column density of interstellar gas and is also the home of potential cosmic-ray acceleration sitesWolf-Rayet stars (van der Hucht 2001), OB associations (Bochkarev \& Sitnik 1985), and supernova remnants (SNRs; Green 2004). The one previously known $\mathrm{TeV}$ source in this region, $\mathrm{TeV}$ J2032+413 (Aharonian et al. 2005; Konopelko et al. 2007), is spatially coincident with the unusual globular cluster OB2 (Knodlseder 2000). The Tibet Air Shower detector also recently reported an excess in the $\mathrm{TeV}$ cosmic-ray flux from this region (Amenomori et al. 2006). In this Letter, we report a detailed analysis of the Milagro $\mathrm{TeV}$ data (with substantially higher sensitivity than our previous work) from the Cygnus region.

\section{DATA ANALYSIS}

The Milagro gamma-ray observatory is a water Cerenkov detector that detects gamma rays above a few times $100 \mathrm{GeV}$ and continuously views the entire overhead sky (Sullivan et al. 2001). The large field of view of $\sim 2$ sr combined with the long observation time of over 5 years makes Milagro the most sensitive instrument available for the study of large, low surface brightness sources such as the diffuse gamma radiation arising from interactions of cosmic radiation with interstellar matter. The Milagro data used in this analysis were collected from 2000 July to 2006 March during which time the average duty factor was greater than $90 \%$. An array of outrigger water tanks was added in 2003 May, which substantially improved the sensitivity of Milagro. The trigger rate of Milagro has remained relatively constant at $\sim 1700$ extensive air showers per second, most of which are due to cosmic-ray-initiated showers. 
The background due to cosmic-ray showers is estimated using the methods described in Atkins et al. (2003); however, a more efficient parameter for distinguishing between gamma rays and cosmic rays has been developed (Abdo 2006, 2007). This parameter, $A_{4}$, is defined as

$$
A_{4}=\frac{\left(f_{\mathrm{top}}+f_{\mathrm{out}}\right) n_{\mathrm{fit}}}{\mathrm{PE}_{\mathrm{bot}}^{\max }}
$$

where $f_{\text {top }}$ and $f_{\text {out }}$ are the fraction of photomultiplier tubes (PMTs) in the top layer of the pond detector and the fraction of outrigger tanks hit, respectively, $n_{\mathrm{fit}}$ is the number of PMTs retained in the angle fit, and $\mathrm{PE}_{\mathrm{bot}}^{\max }$ is the number of photoelectrons in the bottom layer PMT with the most photoelectrons. In the data before the outriggers were installed, $f_{\text {out }}$ is set equal to zero. Figure 1 shows the distributions of $A_{4}$ for proton and gamma-ray Monte Carlo events and for data. The gamma-ray distribution extends to larger values of $A_{4}$ because these showers contain fewer penetrating particles, such as muons and hadrons, which produce localized light in the bottom layer to give large values of $\mathrm{PE}_{\mathrm{bot}}^{\max }$ without greatly increasing the number of hit PMTs.

The signal-to-background ( $\mathrm{S} / \mathrm{B})$ ratio improves as $A_{4}$ values increase. Therefore, instead of counting all the events in an angular bin equally, a weighted sum of events is used in which events with higher values of $A_{4}$ are assigned higher weights. The significance is computed using the method described in $\mathrm{Li} \&$ Ma (1983), where background fluctuations are similarly estimated for the sum of the weights of the background sample, rather than the background event count. The statistical significance derived from this analysis was verified both by Monte Carlo simulation and through the study of statistical fluctuations in the background data sample.

The values of the weights used in this analysis are determined from the predicted $\mathrm{S} / \mathrm{B}$ ratio as calculated a priori from our detector simulation for an incident Crab-like spectrum. With this weighting scheme, this analysis is equivalent to a likelihood ratio method estimation in the limit that the background is large, which is true for the Milagro data. Different weights are used for the data with and without the outriggers and for different experimental configurations of which there are five epochs considered.

The source flux is determined from a Monte Carlo simulation of the detection efficiency of Milagro to an assumed source flux and spectrum that gives the expected excess for each interval in $A_{4}$. The expected counts in each interval are then multiplied by the weight determined from the simulation for that $A_{4}$ interval. The results from each epoch are multiplied by the duration of that epoch, and then the ratio of the observed excess to the sum of the weights from all epochs is multiplied by the assumed source flux to get the observed flux. This method is repeated for all declinations because the effective area of Milagro varies with zenith angle.

The dependence of the derived source flux on the spectrum is minimized when it is quoted at the median-detected energy, which is $\sim 12 \mathrm{TeV}$ for typical gamma-ray power-law spectra and the weighted analysis using $A_{4}$. For the declinations reported in this Letter, a change in the assumed source differential photon spectral index from -2.4 to -2.8 changes the quoted flux at $12 \mathrm{TeV}$ by $<10 \%$. With this technique, the Crab flux at $12 \mathrm{TeV}$ is determined from the observed $14.2 \sigma$ excess in this 5.8 yr time interval to be consistent with the flux measured by the HEGRA atmospheric Cerenkov telescope (Aharonian et al. 2004). However, the Milagro trigger rate as determined from simulations of protons and helium using the flux from direct

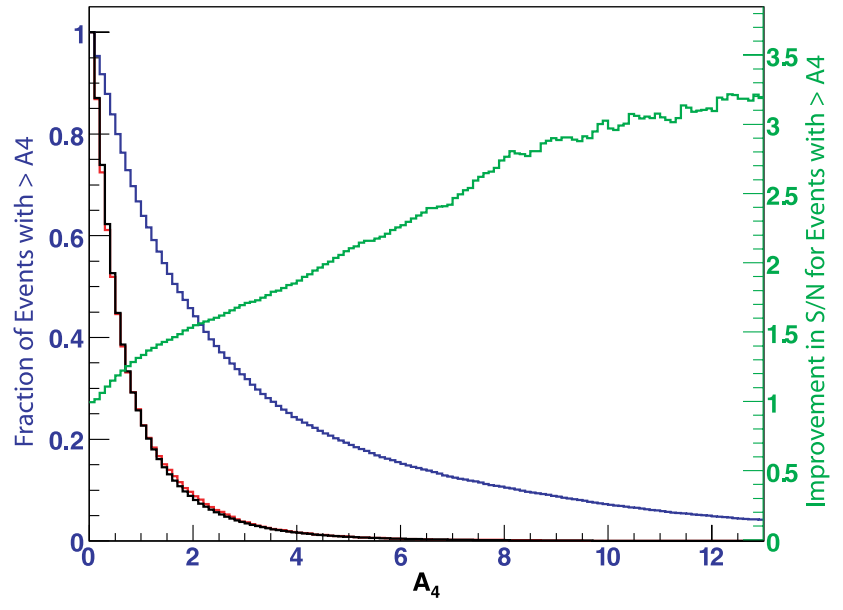

FIG. 1.-Distribution of $A_{4}$, a parameter used to distinguish the cosmic-ray background from the gamma-ray signal. Monte Carlo predictions for cosmicray-initiated showers are shown as a red line, and for gamma-ray-initiated showers are shown as a blue line. The differential particle spectrum for protons is chosen to be a power law of index -2.7 , and for gamma rays it is chosen to be -2.6 . The $A_{4}$ distribution of the background data is shown as a black line. The green line indicates the improvement in the signal-to-noise ratio made by excluding lower values of $A_{4}$.

measurements (Haino et al. 2004; Asakimori et al.1998) is underpredicted, so a systematic error of $30 \%$ is given to the gamma-ray fluxes quoted here.

The weighted analysis enhances the contribution of higher $A_{4}$ events that are, on average, higher energy gamma rays. The median energy with this analysis is $12 \mathrm{TeV}$ for a Crab-like spectrum with a differential photon spectral index of -2.6 . By comparison, a previously published Milagro analysis had a median energy of 3$4 \mathrm{TeV}$. The combination of the installation of the outrigger array, the adoption of the $A_{4}$ discriminant, and the event weighting increases the sensitivity of Milagro as compared to previous analysis by $\sim 2.5$ times as confirmed by observation of the Crab.

\section{RESULTS}

Figure 2 shows of the Cygnus region of the Galactic plane as determined from the Milagro data with this weighted analysis technique. The TeV emission is correlated with the matter density, which is shown as contours in Figure 2, with the exception of a significant excess near Galactic longitude $75^{\circ}$. This new source, MGRO J2019+37, is the second brightest region of $\mathrm{TeV}$ emission (after the Crab Nebula) in the northern hemisphere and is observed with $10.9 \sigma$ excess.

The location of MGRO J2019+37 is R.A. $=304.83 \pm$ $0.14_{\text {stat }} \pm 0.3_{\text {sys }}$ deg and decl. $=36.83 \pm 0.08_{\text {stat }} \pm 0.25_{\text {sys }}$ deg. The systematic error is a combination of the $0.07^{\circ}$ uncertainty in the Milagro location of the Crab, which is used to adjust the absolute pointing of Milagro, and the uncertainties due to the unknown source morphology and to the diffuse background in the Cygnus region. The location of MGRO J2019+37 is consistent (within the combined location errors of EGRET and Milagro) with two EGRET sources. One of the EGRET sources $(3 E G \mathrm{~J} 2016+3657)$ is positionally coincident with the blazarlike source of unknown redshift, B2013+370 (Mukherjee et al. 2000), and the other (3EG J2021+3716) with the young pulsar wind nebula (PWN) G75.2+0.1 (Hessels et al. 2004; Roberts et al. 2002). An analysis of the highest energy photons $(>1 \mathrm{GeV})$ observed by EGRET from this region indicates that the two sources were not resolved by EGRET. A young open 


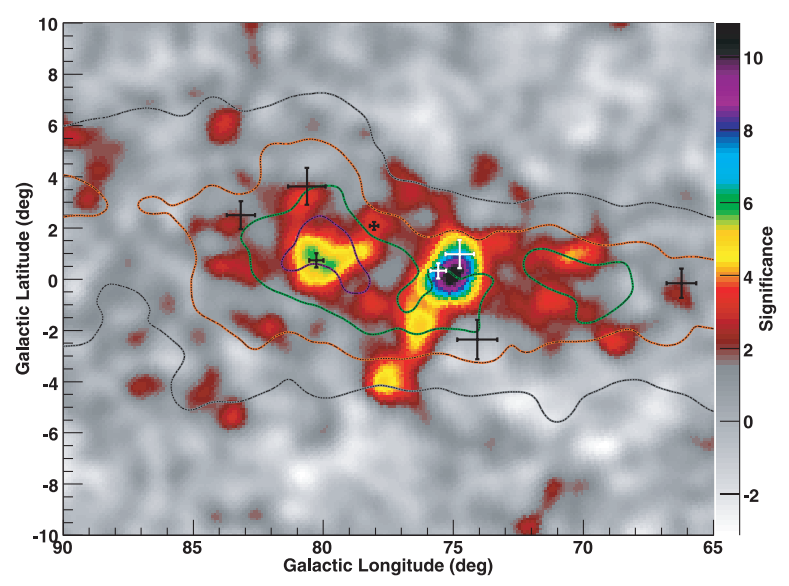

FIG. 2.-Cygnus region of the Galaxy as seen in TeV gamma rays. The statistical significance in units of the standard deviation of the background of the observed excess (or deficit) is plotted. Since the Milagro exposure and sensitivity are roughly constant over the region in the figure, the statistical significance is nearly proportional to the flux from each point. Superimposed on the image are contours showing the matter density (Kalberla et al. 2005; Dame et al. 2001; Leung \& Thaddeus 1992). The crosses show the location of EGRET sources and their corresponding location errors.

star cluster, Berkeley 87, is also nearby and could produce $\mathrm{TeV}$ gamma rays by shocks from interstellar winds as is postulated for Cyg OB2 and TeV J2032+413 (Butt et al. 2006). Berkeley 87 was observed by HEGRA (Aharonian et al. 2006b), and a flux upper limit above $1 \mathrm{TeV}$ was obtained. Given that the median energy of the Milagro observation is $12 \mathrm{TeV}$, a blazar-like source is less likely because such high-energy gamma rays are attenuated by interactions with the extragalactic infrared background.

While the angular resolution of Milagro for an average gamma ray is $0.5^{\circ}$, the highest energy gamma rays detected have substantially better angular resolution $\left(0.35^{\circ}\right)$. An examination of the arrival directions of the higher energy photons shows that MGRO J2019+37 is most likely an extended source or multiple unresolved sources of $\mathrm{TeV}$ gamma rays. A fit of the source to a circular two-dimensional Gaussian, shown in Figure 3, gives $\sigma=0.32^{\circ} \pm 0.12^{\circ}$. A fit with an elliptical two-dimensional Gaussian gives an $\sim 2$ times larger extent in the direction of right ascension than declination. The distance to PWN G75.2+0.1 is estimated to be between 8 and $12 \mathrm{kpc}$ from the dispersion measure of $369 \mathrm{pc} \mathrm{cm}^{-3}$ (Roberts et al. 2002). If MGRO J2019+37 is due to this PWN, then the source radius is $30-90 \mathrm{pc}$. However, if the source lies within the Cygnus region at a distance of 1$2 \mathrm{kpc}$, the source radius is only $4-15 \mathrm{pc}$.

Assuming a differential source spectrum of $E^{-2.6}$, the Milagro flux measurement from a $3 \times 3 \mathrm{deg}^{2}$ bin centered on the location given above for MGRO $\mathrm{J} 2019+37$ is given by $E^{2} d N / d E=$ $\left(3.49 \pm 0.47_{\text {stat }} \pm 1.05_{\text {sys }}\right) \times 10^{-12} \mathrm{TeV} \mathrm{cm}^{-2} \mathrm{~s}^{-1}$ at the mediandetected energy of $12 \mathrm{TeV}$. The diffuse flux from this region is difficult to determine, but as seen in Figure 2 it could be $30 \%-$ $40 \%$ of the total flux. EGRET measured the integral flux above $100 \mathrm{MeV}$ of 3EG $2021+3716$ to be $(59.1 \pm 6.2) \times 10^{-8} \mathrm{~cm}^{-2}$ $\mathrm{s}^{-1}$ with a differential spectral index at $100 \mathrm{MeV}$ of $-1.86 \pm$ 0.10 (Hartman et al. 1999). The flux measured by Milagro above $12 \mathrm{TeV}$ is a factor of 20-300 below an extrapolation of the EGRET spectrum (where the spread accounts for the errors on both the EGRET and Milagro measurements); therefore, if the two sources are the same, the spectrum must exhibit a spectral softening between $100 \mathrm{MeV}$ and $12 \mathrm{TeV}$. A simple power-law

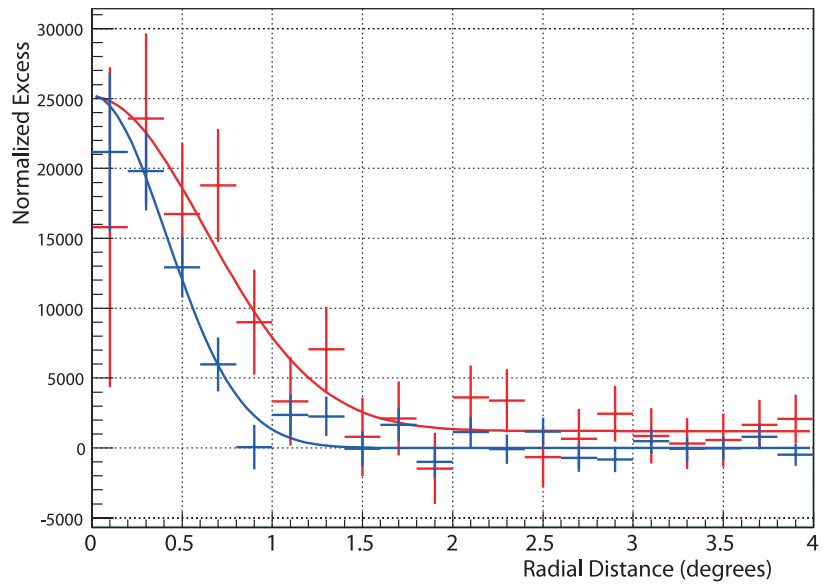

FIG. 3.- Radial profile of events from the direction of the Crab (blue line) and from MGRO $\mathrm{J} 2019+37$ (red line).

fit between the $100 \mathrm{MeV}$ and the $12 \mathrm{TeV}$ flux yields a differential photon spectral index of $-2.22 \pm 0.02$.

The next brightest $\mathrm{TeV}$ region is just to the left of MGRO $\mathrm{J} 2019+37$ in Figure 2, at Galactic latitude of $\sim 80^{\circ}$, and is also coincident with an EGRET source (3EG J2033+4118) and the HEGRA source TeV J2032+413. The HEGRA source was detected between 1 and $10 \mathrm{TeV}$ with a differential photon spectral index of $-1.9 \pm 0.1_{\text {stat }} \pm 0.3_{\text {sys }}$, which when extrapolated to $12 \mathrm{TeV}$ gives $E^{2} d N / d E=\left(7.9 \pm 2.7_{\text {stat }}\right) \times 10^{-13} \mathrm{TeV} \mathrm{cm}^{-2}$ $\mathrm{s}^{-1}$ (Aharonian et al. 2005). The Milagro flux in a $3 \times 3 \mathrm{deg}^{2}$ region centered on the HEGRA source at $12 \mathrm{TeV}$ is $(2.41 \pm$ $\left.0.48_{\text {stat }} \pm 0.72_{\text {sys }}\right) \times 10^{-12} \mathrm{TeV} \mathrm{cm}^{-2} \mathrm{~s}^{-1}$, assuming a differential photon source spectrum of $E^{-2.6}$. Thus, the Milagro flux exceeds the HEGRA flux, as is expected due to the additional contribution of the diffuse flux in this region. In fact, this region contains the largest matter density, as can be seen from the contour lines of Figure 2.

To study the diffuse emission from the Cygnus region, $3 \times$ $3 \mathrm{deg}^{2}$ around MGRO $\mathrm{J} 2019+37$ is excluded from the area defined by Galactic latitude $-3.0^{\circ}$ to $3.0^{\circ}$ and Galactic longitude $65^{\circ}-85^{\circ}$. For the remaining region, the energy flux at $12 \mathrm{TeV}$ is $E^{2} d N / d E=\left(4.18 \pm 0.52_{\text {stat }} \pm 1.26_{\text {sys }}\right) \times 10^{-10} \mathrm{TeV} \mathrm{cm}^{-2} \mathrm{~s}^{-1}$ $\mathrm{sr}^{-1}$, assuming a differential photon source spectrum of $E^{-2.6}$.

\section{CONCLUSIONS}

The expected gamma-ray emissivity due to cosmic-ray interactions with matter is predicted by the GALPROP (Strong et al. 2004a) program. The GALPROP model calculates the gamma-ray emissivities in every spatial grid point using the propagated spectra of cosmic-ray species, leptons and nucleons, the interstellar radiation field, and the gas densities. The gasrelated components (pion decay and bremsstrahlung) of the gamma-ray sky maps are calculated using $21 \mathrm{~cm}$ line survey data for $\mathrm{H}$ I and $\mathrm{CO} J=1$ to $J=0$ survey data for $\mathrm{H}_{2}$, in the form of column densities for galactocentric rings, using velocity information and a rotation curve. The cosmic-ray source distribution is based on SNRs/pulsars and a variable CO-to- $\mathrm{H}_{2}$ conversion factor (Strong et al. 2004b).

The "conventional" model is tuned to have the propagated cosmic-ray particle spectra and intensities match the local direct measurements. This model yields a deficit of diffuse gamma-ray emission above $1 \mathrm{GeV}$, a so-called $\mathrm{GeV}$ excess, observed in all 
directions on the sky. The "optimized" model (Strong et al. 2004a, 2004b) is tuned to match the EGRET diffuse emission data for the whole sky and reproduces the $\mathrm{GeV}$ excess by relaxing the constraints on matching the local cosmic-ray proton and electron measurements. This "optimized" model is instead based on the secondary antiprotons in cosmic rays and EGRET diffuse gamma-ray data, but is independent of the Milagro measurements. In this model the cosmic-ray intensities are significantly higher than those measured locally. Both models are shown in Figure 4 with the EGRET and Milagro measurements.

The Cygnus region is in a direction tangential to our spiral arm located at approximately the same distance from the Galactic center as the solar system. This direction is the most accurate for determining the gas distribution based on velocity information and the Galactic rotation curve. As shown in Figure 4, the Milagro measurement of the diffuse flux in the Cygnus region is a factor of $\sim 7$ above predictions of the "conventional" model. The Milagro flux also exceeds the prediction of the "optimized" model, which incorporates higher cosmic-ray intensities to fit the EGRET data. Increasing the gas column density to agree with the Milagro data would violate the restrictions imposed by the EGRET data, and increasing the cosmic-ray flux at higher energies would violate constraints such as antiproton flux measurements unless the increase in cosmic rays was local to the Cygnus region. Both the parameters of the GALPROP model and the Milagro flux measurement have large systematic uncertainties; however, the discrepancy between the model and the data likely imply the existence of an additional gamma-ray component. The spectrum of this component must be hard-for example, a differential photon spectral index of -2.3 to -2.4 - in order to agree with fluxes measured by both the EGRET and Milagro.

There are several possible explanations for this component: unresolved sources of $\mathrm{TeV}$ gamma rays, a population of highenergy electrons in the region producing an inverse Compton flux at $\mathrm{TeV}$ energies, or a population of dark cosmic-ray accelerators in which the hadrons do not interact near their sources but instead with the local matter distribution. The correlation of the observed emission with the matter density can be consistent with all of these explanations if the sources are colocated with the matter. If the excess is due to inverse Compton scattered photons, then this component must be a factor of $\sim 20$ higher at $12 \mathrm{TeV}$ than the prediction of the conventional model. Given typical diffusion and energy-loss times of such highenergy electrons, the source of these electrons must reside within the Cygnus region. Also, the proposed explanation of the $\mathrm{GeV}$ excess due to neutralino annihilation (de Boer et al.

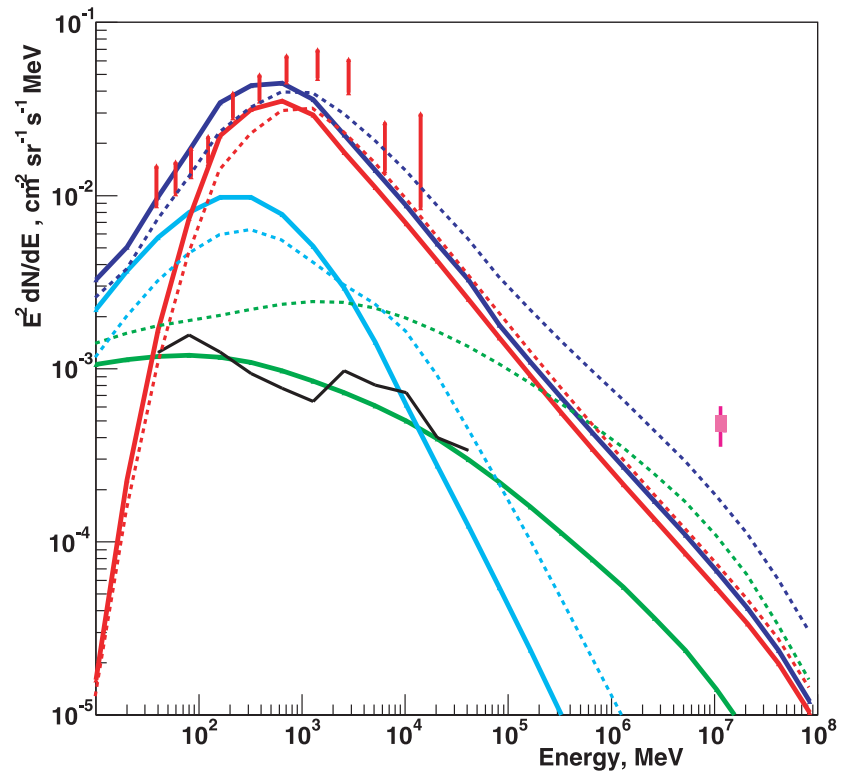

FIG. 4.-Gamma-ray spectrum of the diffuse emission from the Cygnus region of the Galactic plane. The red bars are the EGRET data, and the purple bar is the Milagro measurement with the statistical error shown as a broad line and with the systematic error shown as a narrow line. The solid lines represent the "conventional," and the dotted lines represent the "optimized," GALPROP model of Strong et al. (2004a, 2004b). The dark blue lines represent the total diffuse flux, the red lines represent the pion component, the green lines represent the inverse Compton component, the light blue lines are due to bremsstrahlung, and the black lines are due to the extragalactic background. The Milagro analysis is insensitive to isotropic emission due to the background subtraction, so the extragalactic background is not included in the Milagro energy range.

2005) cannot explain the Milagro high-energy flux, because such a massive neutralino would have a much smaller number density and hence lower flux. Therefore, this Milagro observation suggests that the Cygnus region contains hard-spectrum, cosmic-ray proton or electron accelerators.

The authors thank Seth Digel for his help with the matter density contours. This work is supported by the NSF (PHY0245143, -0245234, -0302000, -0400424, -0504201, and ATM0002744), the DoE (Office of High-Energy Physics and Office of Nuclear Physics), the Los Alamos National Laboratory, the University of California, and the Institute of Geophysics and Planetary Physics. I. V. M. was supported by a NASA APRA grant.

\section{REFERENCES}

Abdo, A. A. 2006, in AIP Conf. Proc. 867, Calorimetry in High Energy Physics:

12th Int. Conf., ed. S. R. Magill \& R. Yoshida (New York: AIP), 199 2007, Ph.D. thesis, Michigan State Univ.

Aharonian, F., et al. 2004, ApJ, 614, 897

2005, A\&A, 431, 197

2006a, Nature, 439, 695

2006b, A\&A, 454, 775

Amenomori, M., et al. 2006, Science, 314, 439

Asakimori, K., et al. 1998, ApJ, 502, 278

Atkins, R., et al. 2003, ApJ, 595, 803

2005, Phys. Rev. Lett., 95, 251103

Bochkarev, N. G., \& Sitnik, T. G. 1985, Ap\&SS, 108, 237

Butt, Y., et al. 2006, ApJ, 643, 238

Dame, T. M., Hartmann, D., \& Thaddeus, P. 2001, ApJ, 547, 792

de Boer, W., Sander, C., Zhukov,V., Gladyshev, A. V., \& Kazakov, D. I. 2005,

A\&A, 444, 51

Gralewicz, P., Wdowcyzk, J., Wolfendale, A. W., \& Zhang, L. 1997, A\&A, 318,925

Green, D. A. 2004, Bull. Astron. Soc. India, 32, 325

Haino, S., et al. 2004, Phys. Lett. B, 59435
Hartman, R. C., et al. 1999, ApJS, 123, 79

Hessels, J. W. T, et al. 2004, ApJ, 612, 389

Hunter, S. D., et al. 1997, ApJ, 481, 205

Kalberla, P. M. W., Burton, W. B., Hartmann, D., Arnal, E. M., Bajaja, E.,

Morras, R., \& Pöppel, W. G. L. 2005, A\&A, 440, 775

Knodlseder, J. 2000, A\&A, 360, 539

Konopelko, A., et al. 2007, ApJ, in press (astro-ph/0611730)

Leung, H. O., \& Thaddeus, P. 1992, ApJS, 81, 267

Li, T. P., \& Ma, Y. Q. 1983, ApJ, 272, 317

Mukherjee, R., et al. 2000, ApJ, 542, 740

Porter, T. A., \& Protheroe, R. J. 1997, J. Phys. G, 23, 1765

Prodanović, T., Fields, B. D., \& Beacom, J. F. 2007, Astropart. Phys., 27, 10

Roberts, M. S. E., Hessels, J. W. T., Ransom, S. M., \& Kaspi, V. M. 2002, ApJ, 577, L19

Strong, A. W., Moskalenko, I. V., \& Reimer, O. 2004a, ApJ, 613, 962

Strong, A. W., Moskalenko, I. V., Reimer, O., Digel, S., \& Diehl, R. 2004b, A\&A, 422, L47

Sullivan, G., et al. 2001, Proc. 27th Int. Cosmic-Ray Conf. (Hamburg), 2773 van der Hucht, K. A. 2001, NewA Rev., 45, 135 